\title{
Ideas and perspectives: Proposed best practices for collaboration at cross-disciplinary observatories
}

\author{
Jason Philip Kaye ${ }^{1}$, Susan L. Brantley ${ }^{2,3}$, Jennifer Zan Williams ${ }^{3}$, and the SSHCZO team* \\ ${ }^{1}$ Department of Ecosystem Science and Management, The Pennsylvania State University, University Park, PA 16802, USA \\ ${ }^{2}$ Department of Geosciences, The Pennsylvania State University, University Park, PA 16802, USA \\ ${ }^{3}$ Earth and Environmental Systems Institute, The Pennsylvania State University, University Park, PA 16802, USA \\ *A full list of authors and their affiliations appears at the end of the paper.
}

Correspondence: Jason Philip Kaye (jpk12@psu.edu)

Received: 25 June 2019 - Discussion started: 9 July 2019

Revised: 21 October 2019 - Accepted: 22 October 2019 - Published: 9 December 2019

\begin{abstract}
Interdisciplinary science affords new opportunities but also presents new challenges for biogeosciences collaboration. Since 2007, we have conducted site-based interdisciplinary research in central PA, USA, at the Susquehanna Shale Hills critical zone observatory. Early in our collaboration, we realized the need for some best practices that could guide our project team. While we found some guidelines for determining authorship on papers, we found fewer guidelines describing how to collaboratively establish field sites, share instrumentation, share model code, and share data. Thus, we worked as a team to develop a best practices document that is presented here. While this work is based on one large team project, we think many of the themes are universal, and we present our example to provide a building block for improving the function of interdisciplinary biogeoscience science teams.
\end{abstract}

\section{Introduction}

Interdisciplinary science has proliferated in recent decades, resulting in larger science teams drawing on increasingly complex research infrastructure (Lattuca, 2001; Rhoten and Parker, 2004; National Academy of Sciences, 2005; Pearce et al., 2010; Hinckley et al., 2016). The scientific community has embraced interdisciplinary research because it leads to discoveries that would not arise from work in isolated disciplines and because many of the most intriguing questions lie at the boundaries of fields. However, science that crosses disciplines and is generated by teams also brings new challenges related to attribution of credit and management of shared infrastructure and data. While social scientists have studied interdisciplinary science (Lattuca, 2001; Rhoten and Parker, 2004), domain scientists themselves have not thoroughly grappled with the problems that arise. As a result, there are relatively few published models or guidelines to promote efficient and collegial management of interdisciplinary science. While guidelines for co-authorship have been published (Weltzin et al., 2006; Oliver et al., 2018), this represents just one of the challenges of interdisciplinary collaboration. Other aspects, such as shared equipment, colocation of equipment, maintenance, sharing samples, and computer codes, etc. are all areas where we lack established guidelines to promote efficient collaboration.

Interdisciplinary work is proliferating throughout the biogeosciences, including research catalyzed recently by the emergence of the critical zone (CZ) approach. Earth's CZ is the thin near-surface zone spanning from bedrock to the atmospheric boundary layer (National Research Council, 2001; Brantley et al., 2007). Since the mid-2000s, scientists have been viewing this zone through a new interdisciplinary lens that brings together biology, soil science, geology, hydrology, and meteorology to make co-located measurements of chemical and biological transport and transformation that describe past landscape evolution and improve projections of future conditions (Brantley et al., 2017; Sullivan et al., 2019). This interdisciplinary approach has precipitated important insights that link hydrology, weathering rates, soil characteristics, nutrient availability, microbial processes, land-air 
fluxes, and plant dynamics (e.g., Hahm et al., 2014; Richter and Billings, 2015).

While interdisciplinary critical zone observatory (CZO) research is attracting great scientific interest and funding opportunities, it also creates problems that most scientists are not trained to address. Disciplinary norms vary in how credit is attributed for publications, data, and model code. In addition, the $\mathrm{CZ}$ approach relies on a wide array of instrumentation that must be deployed systematically, and, again, disciplinary norms vary in how to prioritize instrumentation siting and maintenance. To our knowledge, there are no published guidelines to facilitate the collegial and efficient management of $\mathrm{CZ}$ science in the observatory setting, a facilitation that is integral to long-term site-based scientific studies (National Research Council, 2014). In this paper, we seek to initiate a community-wide discussion about CZ collaboration by sharing our own guide for "best management practices" at our CZO.

\section{The Susquehanna Shale Hills critical zone observatory}

In 2007, the US National Science Foundation (NSF) began funding a network of CZOs (Brantley et al., 2017), and one of these initial CZOs was the Susquehanna Shale Hills critical zone observatory (SSH CZO), where we work. After collaborating for about a decade and discussing ideas in several all-team meetings, we distilled lessons learned from our successes and mistakes into the best practices guide presented below. Ours is not a definitive model but rather a single and evolving example that is meant to foster discussion of how to maximize the benefits of interdisciplinary science. While Sect. 2.1 (co-authorship) has been addressed in some prior publications (Weltzin et al., 2006; Oliver et al., 2018), the major new contributions of this document are the treatment of other aspects of interdisciplinary environmental science, including managing infrastructure, advising students, and sharing model codes and data. Our format is to first lay out the core concepts for key themes (Sect. 2.2, 2.3, 2.4, etc.) and then use a litany of questions and answers to flesh out details for important cases that arise over time. We implement this document by asking CZO scientists to discuss it and sign it in periodic team sessions.

There are some critical components of any team running an environmental observatory that need to be defined. Different observatories may define their core staff in different fashions, but all such observatories must accomplish similar tasks. For example, a program coordinator typically coordinates the personnel and the reporting and team meetings. In addition, our program coordinator is in charge of registering and archiving samples. Typically, a data manager is responsible for managing data that streams from the catchment as well as publication of data from other instrumentation online. Finally, a field operations specialist is needed to manage the field deployment of instrumentation, including the data streaming. This person also works with the program co- ordinator to balance competing needs for space or instrumentation. Similarly, the field operations specialist and program coordinator must coordinate the team in maintaining clean and orderly sites. A director supervises all three of these personnel. The governing body of the SSH CZO is a steering committee described in Sect. 2.7. The home institution for our team is the Pennsylvania State University (abbreviated "Penn State" below). Our CZO includes one large watershed, Shavers Creek $\left(165 \mathrm{~km}^{2}\right)$, as well as three nested subcatchments (Brantley et al., 2017, 2018; Li et al., 2018), each with distinct ownership and permitting (see Sect. 2.2).

\section{Best practices document}

\subsection{Best practices for authorship on peer-reviewed papers}

Our criteria for authorship are based the Ecological Society of America code of ethics (ESA, 2013), and they are consistent with recommendations of the American Geophysical Union Committee on Publication Ethics (Albert and Wagner, 2003). Authorship may be anticipated if researchers make substantial contributions in one or more of the following areas:

1. creation of the conceptual ideas or experimental design,

2. management or execution of the study,

3. analysis or interpretation of data,

4. writing of the manuscript.

We do not prescribe levels for substantial contribution and so each manuscript will require an open discussion regarding authorship. However, to provide some guidance, substantial is taken here to mean a contribution that either involves planning and analysis beyond that available at a commercial laboratory, creative or long-term field work, development of models, or other similar contributions. In general, engagement in writing is often a key delineation of co-authorship. Thus, it is important that scientists contributing to no. 2 (e.g., longterm collection of field data) are sought out and afforded the opportunity to contribute in the analysis and writing stages of the manuscript. It must be recognized that different disciplines have different codes of authorship and so flexibility must be retained. Regardless, the discussion and agreement should be achieved early in the collaboration, and the senior scientist should promote this discussion. In ambiguous cases, we are inclined to err on the side of being more generous with authorship. Once established, authorship and the order of authors shall not be changed without consulting all the authors on the manuscript. No author shall be included on a manuscript that has not agreed to the content in the final version. This means that every author must be given a reasonable amount of time to read revisions of the manuscript, 
but, in turn, if an author does not respond for revisions in a reasonable amount of time, they can also lose co-authorship.

Some questions that have arisen are discussed specifically below.

\subsubsection{If I use someone's old, published data, should they be included as a co-author?}

No, prior publication of data does not, in itself, constitute a significant contribution to new papers.

\subsubsection{If I use someone's old, unpublished data, should they be a co-author?}

If the data are unpublished but also not embargoed, then we encourage the authors to engage the scientist who collected the data at a level that would constitute a substantial contribution. However, if a good faith effort is made to engage the scientist in charge of the original data and that scientist has not responded, then it would not be appropriate to include them as a co-author, but it would be appropriate to acknowledge them and cite the DOI of the dataset. If the old data are embargoed (i.e., not yet public) then the authors must gain permission to use the data. At this time, the two parties (paper authors and data collector) should discuss authorship in the context of the criteria described above. In unusual cases, a researcher who collected embargoed data may not make appropriate progress in publishing a dataset. In that case, the CZO team may need to decide on a course of action with respect to publication of the embargoed data that, in the best case, would involve discussion with the original researcher but might have to proceed without such discussion. Such unusual circumstances should be well-discussed among the steering committee for guidance. A guiding principle is the understanding that scientists have a responsibility to publish data collected with outside funding, whether from federal, state, or foundation sources. Ultimately, a researcher who makes a substantial contribution to a manuscript should be included as a co-author on a publication.

\subsubsection{If I use someone's code or model output from a previously published paper, should they be included as a co-author?}

No, unless the code developer is intellectually engaged in the manuscript development. A couple of examples that might lead to authorship could be that (1) the code developer provides new model outputs and is engaged in output analysis or (2) the code developer runs new model simulations for the manuscript (i.e., performs new calibration, collects new driver data) or adds new functionalities to the model.

\subsubsection{If I use someone's code that has not been published in a paper, should they be included as a co-author?}

Similar to using someone's unpublished data, we encourage the authors to engage the code developer at a level that would constitute a substantial contribution.

\subsubsection{If I collect field samples for someone should I expect to be a co-author on their paper?}

Field sampling is often an overlooked component of the creative environmental science process where critical decisions are made that affect the quality and value of the data. However, field sampling alone is not a contribution that automatically warrants co-authorship. We encourage discussions that enable people who have contributed substantially to field work to become engaged in analysis and writing at a level that warrants co-authorship. The long-term nature or difficulty of field collection can also be taken into account.

\subsubsection{If I test an idea from a CZO proposal, should the principal investigators (PIs) be co-authors on the paper?}

This is a tricky question and varies from one team to another. For example, in some observatory teams, every paper that is published includes the name of the principal investigator. At the SSH CZO, the answer to this question depends on how specific the idea is and how much input the PI has had on the project and the paper. If the authors of the proposal conceived of the idea and described an experimental design to test it, then they may have met criterion no. 1 for co-authorship, and they should be given the opportunity to meet other criteria for co-authorship. However, at our CZO, if the research is not tied to hypotheses that are described in the proposal, then the proposal PIs are not included as authors simply because of their status on the proposal. In addition, PIs may not have generated every hypothesis in the proposal: some work that is accomplished may thus not warrant PI authorship.

\subsubsection{If an undergraduate researcher collected some of the data, should they be a co-author?}

Undergraduate researchers should be considered for authorship under the same criteria as other scientists. We should promote co-authorship in this regard by giving research interns opportunities to contribute to data analysis and writing if the student is ready for such efforts and remains with the team for a sufficient amount of time. However, in some cases, a worker may only do "what is told" and not participate in planning or thinking about the results in any substantial way: in these cases, inclusion as a co-author may not be warranted. 


\subsubsection{How long should co-authors have to review a manuscript?}

Co-authors should discuss timelines for each manuscript. However, a reasonable expectation is that co-authors will read a draft within 1 month of receiving it, assuming that the author has established some sort of reasonable timeline with respect to vacations, trips, etc. Shorter turnaround times may be appropriate for revisions, but co-authors are still expected to read the final (revised) version.

\subsubsection{What do I do if I try repeatedly but I cannot get a co-author to read the manuscript?}

An appropriate approach is the following. When the author finishes a version of the manuscript, he or she discusses with the possible co-authors a timeline or sequence of review (in other words, the authors must have some ability to frame up the timeline - it is not just at the discretion of the first author). If a potential co-author does not read or comment appropriately on a manuscript, the author should propose a reasonable deadline and write in an email, "we will submit this paper without your name unless you read it and comment on it by such and such date: we prefer to retain you as co-author, but we must move forward". In case the potential co-author still does not respond, it is appropriate to remove the potential coauthor from the authorship list. All attempts should be made for other authors to contact the co-author by multiple means (e.g., email and phone) and make it clear that they will be removed from the authorship list if they do not respond in a specified amount of time.

\subsubsection{Who will decide the final author list in cases of contention?}

We expect co-authors to handle this problem in a collegial way. Best practice will always dictate that the discussion of co-authorship be initiated early in the process and be continued throughout the process. The senior scientist on each project should guide this process along. Guidance can also always be sought from the CZO steering committee and the director of the observatory.

\subsubsection{How is the order of authors determined?}

Best practice would be for all the co-authors to decide this in a collegial way; in most cases, the senior author will decide the order of authors. Order of authors is particularly sticky in some cases because different disciplines view author order differently. On the other hand, these differences can also lead to easy choices. For example, in some disciplines first authorship is the most highly regarded position; however, in chemical and life sciences the senior author is often listed last and that is considered a prestigious position as well (Sauermann and Haeussler, 2017). In general, the person who frames and writes the paper should be first author.

\subsubsection{Who should be the corresponding author on a paper?}

It has been our experience that even larger differences in opinion are present among scientists from different disciplines with respect to corresponding author. To some scientists, the corresponding author is simply the lead author of the paper. To others, the corresponding author should be the author who conceived the project, procured funding for the project, is in a stable career position, and would be most likely to be easy to reach for future correspondence. Often, the lead author may be unwilling, unprepared, or unavailable to field questions from the journal and future readers of the paper, and it may be appropriate to assign a co-author to be the corresponding author. To some scientists, it is considered excellent training for $\mathrm{PhD}$ students to be corresponding authors on papers when they are the lead author. The question of assignment of corresponding author is also of note, in that for some junior scientists from specific countries, this assignment carries great weight. Best practices here must again rely on engagement and conversation early in the planning of the paper.

\subsubsection{How can we remember to include all the appropriate co-authors?}

In highly interdisciplinary and large teams, it is not uncommon that an author prepares a paper and forgets to include appropriate co-authors that made significant contributions early in the project. This has happened several times at the SSH CZO and led us to institute a policy whereby every authorship team that starts to put together a paper is asked to share the proposed title, topic, and author list with the program coordinator early in the writing process. The program coordinator then shares the information with the observatory director and an email is sent out to the rest of the team asking if anyone thinks that they should be on the paper as a co-author or if they think they have a significant contribution to make to the paper. Again, discussion can then ensue to decide on authorship and order.

\subsubsection{Does everyone in the team have to agree with everything that is written in every paper?}

Again, this can be a tricky problem in interdisciplinary science. In general, we have experienced many instances wherein project members did not agree on interpretations of data: amicable collaborations were nonetheless pursued and papers published. We encourage ample discussion among the team to learn from one another in such cases. In many cases, co-authors may not entirely agree with every interpretation in a paper; however, the main interpretations should be derived from consensus, and the senior author should make every attempt to discuss and write consensus language that can be agreed upon by the authorship team. 


\subsubsection{When "CZO team" is a co-author on a paper, how is membership determined?}

This is evaluated on a case-by-case basis, but the current paper provides an illustrative example. We felt that team efforts to initially define key elements of a best practices document, followed by annual team discussions of both ideas and text, along with team editing, collectively represented a level of input that warranted authorship for the CZO team, though not necessarily for any individual team member (except for the current named co-authors). We decided that all team members who had participated in initial framing, annual discussions, or team editing would be able to "opt in" as members of the CZO team co-author. We actively tracked down members who had moved on from CZO science to enable them to opt in.

\subsection{Best practices for installing infrastructure or experiments}

Environmental observatories are scientific assets because they provide long-term site-based data; at the same time, these characteristics bring significant challenges (National Research Council, 2014). Best practices for installation of infrastructure require not only careful consideration of impacts on the environment but also on existing infrastructure, needs of other team members, permitted use, and fees. Scientists (including CZO PIs) that would like to initiate new work that is co-located within the bounds of the CZO must propose each idea for installation with the steering committee, the program coordinator, and the field operations specialist, and each installation is typically described for the entire team for comment. As scientists outside the initial team begin to propose work in the site, the observatory director identifies key CZO scientists who must be consulted regarding the new project. PIs are encouraged to share the information with all students in the lab group so potential impacts can be considered. A second email should be sent prior to the installation of the new equipment. If the new research includes destructive sampling or activity that could affect many projects, then the steering committee might present the proposed work in an all-hands meeting to discuss the viability of the new project. If there are conflicting deployments, then the steering committee has the responsibility to determine whether new installations should go forward.

The field operations specialist should be included in both preliminary and developing conversations regarding new equipment. The final placement of all new field infrastructure must be approved by the field operations specialist. Materials that will stay in the field are marked with a PI-specific color using paint, tape, flagging, or some other permanent coloring. Metal tags stamped with identification are often used. Color coding is managed directly with the field operations specialist and the program coordinator. Immediately after installation of new equipment it is a best practice to take a pho- tograph of the installation and share it, by email, with the entire team. In addition, the location of the instrument must be communicated to the data manager who can update maps of equipment.

The SSHCZO currently has usage agreements for each intensively studied subcatchment: the Penn State Forest Lands Office for the Shale Hills subcatchment, Pennsylvania Department of Conservation of Natural Resources (PA DCNR) for the Garner Run subcatchment, and a private landowner for Cole Farm. In all cases, outside-funded projects that are to be co-located with CZO infrastructure must gain separate permits and approval from both the land owner and the CZO steering committee. In general, it is best to initiate discussions with the CZO steering committee, followed by discussions with the landowner. Projects are generally not considered to be under the CZO umbrella by default, although they may eventually be placed there. Permitting requirements vary significantly among land-owner types. For example, every person who works in the Garner Run subcatchment as part of the CZO (student and faculty advisor, inside or outside of Penn State) must sign the agreement with the PA DCNR and this must be kept on record by the program coordinator. If a PI initiates new work in the area that is not listed in our permit, a new permit must be negotiated directly between the PI and the DCNR and recorded by the program coordinator. It can take up to 3 months for the permit process with the DCNR. If work is pursued in these lands without signing the form or if work is pursued which is not described on the agreements, the $\mathrm{CZO}$ will rescind permission to work on the project and will work with DCNR to rectify the situation.

In some cases, observatories may include private land or land enabling specific land use practices. For example, our observatory work includes one subcatchment on a practicing farm (Li et al., 2018). In this case, the field operations specialist was designated as the point person for all communication with the landowner, working closely with the land owner and farmer (two separate people). Likewise, when sampling the main stem of the stream throughout the watershed, every CZO worker only accesses public land or asks for permissions to step on private land before sampling. Some private landowners have refused permission for access, and this lack of access is strictly observed. One benefit of working on private land is that $\mathrm{CZO}$ workers can sometimes interact with the landowner and farmer, and every attempt has been made to learn from them as well as to give them information in return.

When a PI receives new funding for new instrumentation (separate from the CZO grant), the CZO itself will not become responsible for the new infrastructure that is emplaced in the CZO catchments. Likewise, the new PI will be encouraged to use the CZO's data infrastructure for publication of data; however, the CZO will not become responsible for the data from the new project nor will the CZO police publication of the new data. Ultimately, however, it is recognized that the PI is generally co-locating the experiment at a CZO 
catchment due to the preexisting research and infrastructure. Given this "value added" by the CZO, the CZO steering committee and field operations specialist can ultimately decide whether certain activities are pursued in the CZO catchments. For example, a proposal might be funded to geophysically monitor Shale Hills that results in a new permitting fee. After initiation of the work, the PI of the new proposal might decide he or she wants to perform trenching up the middle of the catchment. If the CZO steering committee decides this is inappropriate, then the new PI will not be enabled to trench. In this regard, the steering committee will work closely with the landowner to maintain appropriate activity.

\subsection{Best practices for using, maintaining, and sharing existing field infrastructure}

Each PI is responsible for maintaining and promoting collaborative use of the equipment assigned to them via color coding (see Sect. 2.2). While the color codes denote the PI in charge, they do not denote ownership of equipment. All CZO field infrastructure and data are shared. However, no field equipment should be used without first notifying the PI in charge and establishing the terms of use and collaboration. Shared use and collaboration are expected and, in some cases, this may mean developing a plan of collaboration that could lead to co-authorship if the criteria in Sect. 2.1 are met. If PIs cannot agree on terms of shared use, then they should bring the issue to the steering committee. While shared use is the overarching goal, there may be some equipment for which shared use is not appropriate. For example, some cases might involve equipment that is very sensitive, difficult to maintain, expensive, rented, or borrowed. These can be handled on a case by case basis.

The PI in charge may decide that it is best not to maintain equipment in working order, even though the equipment can remain in the field for future activities. For example, lysimeters can stay in place for years without being sampled. In these cases, the PI in charge should notify the field operations specialist and any co-PIs that have used the equipment in the past. A new PI may want to initiate the use of that instrumentation. In that case, the new PI and the original PI will be considered in charge of the equipment and its use. Any time infrastructure is moved or removed, the person in charge should contact the data manager to report the equipment, PI, geolocation data, and the date of change. The following are answers to common questions that may arise during this process.

\subsubsection{What if I cannot maintain the equipment myself?}

There are cases in which the CZO support staff or collaboration among co-PIs may be required to maintain field infrastructure. These will need to be handled on a case by case basis with consideration of the availability of support staff time. In general, when a PI begins a sub-project that will re- quire time from support staff, that requirement must be vetted through the steering committee. The field operations specialist will generally be the person to help in maintenance.

\subsubsection{What if an investigator is not maintaining critical equipment in a way that promotes shared use?}

In these cases, a broader discussion may be needed in which the team may decide to transfer maintenance responsibilities to a different investigator or to allocate more project resources (support staff time or funds for maintenance) to the equipment.

\subsection{Best practices for removing field infrastructure}

If field infrastructure has reached the end of its useful life it should be removed by the PI in charge, as denoted by the color coding, and the landscape returned to original form. There may also be cases in which the equipment is still functional but the PI wants to remove the equipment to reduce the maintenance burden. Before removing equipment for any reason, the PI should work with the field operations specialist to email the CZO team (all co-PIs plus support staff) to determine whether the removal will affect other users.

When the CZO ceases to be funded adequately, or when a sub-project ends, each PI has the responsibility to remove equipment with their color code or negotiate a new use agreement with the landowner. Even before a sub-project ends, the steering committee may decide that equipment must be removed by the field operations specialist (instead of by the PI) because the investigator is not completing necessary maintenance. Our current CZO use agreements stipulate that we will restore the landscape to a pristine condition when we are finished with the project. Each year we also host a watershed cleanup day to pick up litter and maintain the infrastructure.

\subsection{Best practices for collecting, sharing, and archiving samples}

Before going to the field to collect samples, scientists should make the field operations specialist and/or program coordinator aware of their sampling schedule. A best practice is to do this via quarterly planning solicited by email. Sampling protocols should be posted on the CZO shared data space (a specific storage space should be defined) so that all future users can use the same sampling protocol or deviate intentionally. CZO workers should attempt to share samples so that multiple analyses are conducted on the same sample. The scientists sharing the samples should agree on the terms of the collaboration, including the potential for co-authorship.

Every solid and liquid sample collected from the field should become archived if sufficient sample is available and if it is likely or possible that future users might want to access this sample. The program coordinator is responsible for sample archiving. PIs and their students and postdocs should consult with the program coordinator prior to collecting any 
samples so that the archive protocol can be established. The $\mathrm{CZO}$ has an established location for dry storage for solid and water samples. No archive is available for frozen samples. All samples must be registered with an International Geo Sample Number (IGSN) (http://www.geosamples.org/ igsnabout, last access: 28 November 2019) prior to archival. CZO personnel should attempt to share archived samples with one another and with the broader scientific community. Scientists who want to use archived samples are required to contact the principal investigator and describe how the sample will be used. The program coordinator is responsible for facilitating this communication and sharing. Often it is best to discuss the terms of collaboration before the archived sample is released. However, in cases when the collector cannot be consulted or does not consent to the release, the case can go the steering committee. If archive sample retrieval becomes overly time-consuming, arrangements may need to be made to pay someone to find samples. The following are answers to common questions that may arise during this process.

\subsubsection{What if I want to deviate from the established CZO sampling protocol?}

We expect this to happen. A rationale should be provided for the change, and methodologies should be noted in protocols maintained in the shared data storage space so that others will know how and why the change was made. The program coordinator will facilitate and oversee modifications to the protocols.

\subsubsection{What if there is only a little bit of an archived sample left and someone wants to use it up?}

If the collector and user of the archived sample and PI of the CZO agree that this is a good use of the sample, then it can be used. In general, however, samples should not be used up. If there is disagreement, then the steering committee can be consulted.

\subsection{Best practices for sharing data}

Guidelines for sharing CZO data are outlined here: http: //criticalzone.org/national/data/access-czo-data-1national/ \#DataUseAgreement (last access: 28 November 2019). Where possible, a PI should get a DOI for datasets for future citation. In general, we advise that data be stored in the CZO data infrastructure, even for data funded by entities outside of Penn State NSF CZO funds. However, the CZO does not become responsible for archiving these data. Some data sharing will occur prior to uploading the data to the CZO database. Data sharing at this early stage is encouraged and even necessary to enable students and PIs to conduct multidisciplinary research. The parties involved should establish authorship and use expectations at the time the data are shared.
It is a best practice not to directly share your copies of data with third parties. For example, if you have a Microsoft Excel spreadsheet of data that another student or PI has shared, you should not share those data with a third scientist. Instead, it is best to have that scientist access the data by going directly to the $\mathrm{CZO}$ web page or contacting the original data source (PI and student) directly. Under some circumstances (e.g., when you have manipulated data in a way that is beneficial to the third party) you may need to pass on someone else's data to a third party. In that case, you should obtain written consent from the original data source, for example through an email exchange that includes a discussion of terms of authorship and use.

\subsection{Best practices for project management}

The steering committee shall be representative of the team. For example, it can be comprised of a subset of the PIs, staff, and students, with fixed and rotating members. The steering committee should send out updates after their meetings to keep co-PIs appraised of key decisions. The steering committee is an appropriate outlet for all grievances related to the project. Discussions of sensitive issues (e.g., personnel) need not be shared, but decisions regarding allocation of resources and discussions about important changes affecting PIs should be shared. In general, the $\mathrm{CZO}$ management will make every attempt to promote (i) collegiality, (ii) open communication, (iii) excellence in research, (iv) excellence in education, (v) excellence in collaborative science, and (vi) excellence in outreach to the public.

As new PIs become involved in the CZO, the steering committee and all of the PIs will make every attempt to avoid multiple groups working on the same problem. However, some overlap will undoubtedly happen and is appropriate in some cases. The steering committee will thus try to steer PIs to overlap collaboratively or at least collegially while testing alternate hypotheses or methodologies for understanding the functioning of the $\mathrm{CZO}$. The $\mathrm{CZO}$ management ultimately has no authority to prohibit publication of ideas, data, or models for the $\mathrm{CZO}$ and in fact encourages competing ideas, data, and models.

A field crew (generally a team of rotating students, postdocs, and staff supported by the project) will assist with sample collection and general maintenance at the site and will help ensure that field sampling can always be conducted in pairs.

\subsection{Best practices for advising students}

In general, graduate and postdoctoral students who work at the $\mathrm{CZO}$ should be encouraged to co-author joint publications as appropriate. Generally, a student will be first author on the project they spearhead (if they do most of the work and most of the writing), unless they do not move forward on publication in a timely manner. When students do not move 
forward on a project within 1 year of completion of their degree, the PI may write the paper and consider becoming first author.

It is the responsibility of PIs on the CZO to mentor their students regarding $\mathrm{CZO}$ best practices. Having a student sign this document is not enough; continuous mentoring regarding ethics and best practices is expected. PIs are expected to be aware of which data and models their students are using, as well as which datasets originated from other CZO students or PIs, and to be engaged in all discussions regarding authorship and use of data, models, and infrastructure. Furthermore, PIs are expected to share relevant emails with their students including those related to infrastructure and site maintenance.

\subsection{Best practices for outreach}

The CZO has a commitment to complete outreach to nonscientists and the public in general. It is expected that everyone who works at the $\mathrm{CZO}$ will at some time (e.g., once per year) participate in public outreach coordinated by the CZO. However, appropriate clearances are often required before Penn State faculty and staff can participate in outreach with certain populations (e.g., underage students).

\subsection{Best practices for reporting}

It is expected that everyone working at the $\mathrm{CZO}$ will provide reports of effort to the program coordinator in a timely manner. Lack of participation in reporting, if egregious, can be grounds for termination of collaboration at the CZO. Everyone working at the $\mathrm{CZO}$ will also be expected to cite the CZO appropriately (as indicated on the CZO website) and to provide copies of submitted, in press, and published papers to the program coordinator at the time of submission, acceptance for publication, or publication, respectively. Acknowledgements must be written to recognize the changing nature of funding that typically accompanies an observatory, including the host university or other entity.

\section{Conclusion}

There is growing evidence that collaborative teams advance science in distinct ways from individual investigators (Uzzi et al., 2013). This may occur because each PI brings deep but often conventional understanding of their knowledge domain into innovative combinations with collaborators from other domains. Evidence also shows that observatories provide long-term data that enrich our understanding of environmental processes (National Research Council, 2014). Teams can only leverage these innovative ideas and sitespecific data into generalizable models if they work well together through collegial and efficient use of field sites, instrumentation, samples, data, and model code. One key step is agreeing on the best practices for collaboration. Once per year at a project meeting, we discuss best practices, as outlined above, with the entire team of the Susquehanna Shale Hills critical zone observatory. These discussions typically bring to light challenges that are then added to our living best practices document (updated versions posted here: https://doi.org/10.26207/d7ea-v862, last access: 28 November 2019). Most senior scientists at our CZO were not taught to work in teams; thus, developing a code for best practices has helped experienced and young scientists alike to grow an understanding for efficient ways to collaborate. We offer this document as an example, with the hope that it will foster discussion enabling the field of biogeosciences to fully capitalize on large-team collaborative science.

Data availability. No data sets were used in this article.

Team list. David Eissenstat (Dept. of Ecosystem Science and Management, Penn State University, USA), Kenneth Davis (Dept. of Meteorology and Atmospheric Science, Penn State University, USA), Li Li (Dept. of Civil and Environmental Engineering, Penn State University, USA), Tess Russo (Dept. of Geosciences, Penn State University, USA), Roman DiBiase (Dept. of Geosciences, Penn State University), Henry Lin (Dept. of Ecosystem Science and Management, Penn State University, USA), Margot Kaye (Dept. of Ecosystem Science and Management, Penn State University, USA), Yuning Shi (Dept. of Ecosystem Science and Management, Penn State University, USA), Li Guo (Dept. of Ecosystem Science and Management, Penn State University, USA), Elizabeth Hasenmueller (Dept. of Ecosystem Science and Management, Penn State University, USA), Kristen Brubaker (Environmental Studies, Hobart and William Smith Colleges, USA), Thomas Adams (Dept. of Ecosystem Science and Management, Penn State University, USA), Chen Bao (Dept. of Energy and Mineral Engineering, Penn State University, USA), Joanmarie Del Vecchio (Dept. of Geosciences, Penn State University, USA), Xin Gu (Dept. of Geosciences, Penn State University, USA), Yuting He (Dept. of Meteorology and Atmospheric Science, Penn State University, USA), Elizabeth Hoagland (Dept. of Geosciences, Penn State University, USA), Warren Reed (Dept. of Ecosystem Science and Management, Penn State University, USA), Ismaiel Szink (Dept. of Ecosystem Science and Management, Penn State University, USA), Julie Weitzman (Dept. of Ecosystem Science and Management, Penn State University, USA), Dacheng Xiao (Dept. of Energy and Mineral Engineering, Penn State University, USA), Brandon Forsythe (Earth and Environmental Systems Institute, Penn State University, USA), Benjamin Dillner (Dept. of Ecosystem Science and Management, Penn State University, USA), Caitlin Hodges (Dept. of Ecosystem Science and Management, Penn State University, USA), Virginia Marcon (Dept. of Geosciences, Penn State University, USA), Edward Primka IV (Dept. of Ecosystem Science and Management, Penn State University, USA), Perri Silverhart (Dept. of Geosciences, Penn State University, USA), and Qicheng Tang (Dept. of Ecosystem Science and Management, Penn State University, USA). 
Author contributions. JPK wrote the initial draft and is the principal and corresponding author of this living document built from ideas generated and discussed throughout the project. SLB conceived of the idea of this best practices document and contributed to writing. JZW provided logistical leadership and support in the implementation and documentation of these best practices, including soliciting and synthesizing team input. The team provided insightful contributions to the challenges experienced and ideas for practical application of resolution.

Competing interests. The authors declare that they have no conflict of interest.

Acknowledgements. We would like to thank Christopher Duffy for his leadership during the first Susquehanna Shale Hills critical zone observatory grant.

Financial support. This research has been supported by the US National Science Foundation (grant nos. EAR-0725019, EAR1239285 and EAR-1331726).

Review statement. This paper was edited by Jack Middelburg and reviewed by Justus van Beusekom and Sybil Seitzinger.

\section{References}

Albert, T. and Wager, E.: How to handle authorship disputes: a guide for new researchers, COPE, https://doi.org/10.24318/cope.2018.1.1, 2003.

Brantley, S. L., Goldhaber, M. B., and Ragnarsdottir, K. V.: Crossing Disciplines and Scales to Understand the Critical Zone, Elements, 3, 307-314, https://doi.org/10.2113/gselements.3.5.307, 2007.

Brantley, S. L., McDowell, W. H., Dietrich, W. E., White, T. S., Kumar, P., Anderson, S. P., Chorover, J., Lohse, K. A., Bales, R. C., Richter, D. D., Grant, G., and Gaillardet, J.: Designing a network of critical zone observatories to explore the living skin of the terrestrial Earth, Earth Surf. Dynam., 5, 841-860, https://doi.org/10.5194/esurf-5-841-2017, 2017.

Brantley, S. L., White, T., West, N., Williams, J. Z., Forsythe, B., Shapich, D., Kaye, J., Lin, H., Shi, Y., Kaye, M., Herndon, E., Davis, K. J., He, Y., Eissenstat, D., Weitzman, J., DiBiase, R., Li, L., Reed, W., Brubaker, K., and Gu, X.: Susquehanna Shale Hills Critical Zone Observatory: Shale Hills in the Context of Shaver's Creek Watershed, Vadose Zone J., 17, 180092, https://doi.org/10.2136/vzj2018.04.0092, 2018.

ESA: Code of Ethics for the Ecological Society of America, available at: https://www.esa.org/about/code-of-ethics/ (last access: 21 June 2019), 2013.

Hahm, W. J., Riebe, C. S., Lukens, C. E., and Araki, S.: Bedrock composition regulates mountain ecosystems and landscape evolution, P. Natl. Acad. Sci. USA, 111, 3338-3343, 2014.

Hinckley, E. S., Anderson, S. P., Baron, J. S., Blanken, P. D., Bonan, G. B., Bowman, W. D., Elmendorf, S. C., Fierer, N., Fox, A. M.,
Goodman, K. J., Jones, K. D., Lombardozzi, D. L., Lunch, C. K., Neff, J. C., SanClements, M. D., Suding, K. N., and Wieder, W. R.: Optimizing Available Network Resources to Address Questions in Environmental Biogeochemistry, BioScience, 66, 317 326, dio:10.1093/biosci/biw005, 2016.

Lattuca, L. R.: Creating Interdisciplinarity: Interdisciplinary Research and Teaching among College and University Faculty, Vanderbilt University Press, Nashville, TN, 2001.

Li, L., DiBiase, R., Del Vecchio, J., Marcon, V., Hoagland, B., Xiao, D., Wayman, C., Tang, Q., He, Y., Silverhart, P., Szink, I., Forsythe, B, Williams, J. Z., Shapich, D., Mount, G., Kaye, J., Guo, L., Lin, H., Eissenstat, D., Dere, A., Brubaker, K., Kaye, M., Davis, K. J., Russo, T., and Brantley, S. L.: The Effect of Lithology and Agriculture at the Susquehanna Shale Hills Critical Zone Observatory, Vadose Zone J., 17, 180063, https://doi.org/10.2136/vzj2018.03.0063, 2018.

National Academy of Sciences: Facilitating Interdisciplinary Research, The National Academies Press, Washington, DC, https://doi.org/10.17226/11153, 2005.

National Research Council: Basic Research Opportunities in Earth Science, The National Academies Press, Washington, DC, https://doi.org/10.17226/9981, 2001.

National Research Council: Enhancing the Value and Sustainability of Field Stations and Marine Laboratories in the 21st Century, Washington, DC, The National Academies Press, https://doi.org/10.17226/18806, 2014.

Oliver, S. K., Fergus, C. E., Skaff, N. K., Wagner, T., Tan, P., Cheruvelil, K. S., and Soranno, P. A.: Strategies for Effective Collaborative Manuscript Development in Interdisciplinary Science Teams, Ecosphere, 9, e02206, https://doi.org/10.1002/ecs2.2206, 2018.

Pearce, A. R., Bierman, P. R., Druschel, G. K., Massey, C., Rizzo, D. M., Watzin, M. C., and Wemple, B. C.: Pitfalls and Successes of Developing an Interdisciplinary Watershed Field Science Course, Journal of Geoscience Education, 58, 145-154, https://doi.org/10.5408/1.3544295, 2010.

Rhoten, D. and Parker, A.: Risks and Rewards of an Interdisciplinary Research Path, Science, 306, 2046, https://doi.org/10.1126/science.1103628, 2004.

Richter, D. de B. and Billings, S. A.: "One physical system": Tansley's ecosystem as Earth's critical zone, New Phytol., 206, 900912, https://doi.org/10.1111/nph.13338, 2015.

Sauermann, H. and Haeussler, C.: Authorship and Contribution Disclosures, Science Advances, 3, e1700404, https://doi.org/10.1126/sciadv.1700404, 2017.

Sullivan, P. L., Goddéris, Y., Shi, Y., Gu, X., Schott, J., Hasenmueller, E. A., Kaye, J. P., Duffy, C., Lin, H., and Brantley, S. Exploring the effect of aspect to inform future earthcasts of climate-driven changes in weathering of shale, J. Geophys. Res.Earth, 124, 974-993, https://doi.org/10.1029/2017JF004556, 2019.

Uzzi, B., Mukherjee, S., Stringer, M., and Jones, B.: Atypical Combinations and Scientific Impact, Science, 342, 468-472, https://doi.org/10.1126/science.1240474, 2013.

Weltzin, J. F., Belote, R. T., Williams, L. T., Keller, J. K., and Engel, E. C.: Authorship in ecology: attribution, accountability, and responsibility, Front. Ecol. Environ., 4, 435-441, https://doi.org/10.1890/15409295(2006)4[435:AIEAAA]2.0.CO;2, 2006. 